\title{
Constraint qualifications in linear vector semi-infinite optimization*
}

\author{
M.A. Goberna $†$ F. Guerra-Vazquez; and M.I. Todorov ${ }^{\ddagger}$
}

July 16, 2012

\begin{abstract}
Linear vector semi-infinite optimization deals with the simultaneous minimization of finitely many linear scalar functions subject to infinitely many linear constraints. This paper provides characterizations of the weakly efficient, efficient, properly efficient and strongly efficient points in terms of cones involving the data and Karursh-Kuhn-Tucker conditions. The latter characterizations rely on different local and global constraint qualifications. The global constraint qualifications are illustrated on a collection of selected applications.
\end{abstract}

Keywords: Multiple objective programming; Linear vector semi-infinite optimization; Constraint qualifications; Cone conditions; KKT conditions

\section{Introduction}

Linear vector semi-infinite optimization (VSIO in brief) deals with optimization problems of the form

$$
P: " \min " f(x)=\left(c_{1}^{\prime} x, \ldots, c_{p}^{\prime} x\right) \text { s.t. } a_{t}^{\prime} x \geq b_{t}, t \in T,
$$

where $c_{i} \in \mathbb{R}^{n}$ (the space of decisions), $i=1, \ldots, p,\left(a_{t}, b_{t}\right) \in \mathbb{R}^{n+1}$ (the space of coefficients) for all $t \in T$, and the index set $T$ is infinite. When $T$ is finite, $P$ is a linear vector optimization (VO) problem and when $p=1$ and $T$ is infinite, $P$ is a linear semi-infinite optimization (SIO) problem. We say that $P$ is continuous when the index set $T$ is a compact Hausdorff topological space and the coefficient functions, $t \mapsto a_{t}$ and $t \mapsto b_{t}$, are continuous on $T$.

${ }^{*}$ This research was partially supported by MICINN of Spain, Grant MTM2011-29064-C0302 and CONACYT of Mexico, Grant 55681. The first author is Partner Investigator in the Australian Research Council Discovery Project DP120100467.

${ }^{\dagger}$ Corresponding author. Dep. of Statistics and Operations Research, Alicante University, 03071 Alicante, Spain. E-Mail: mgoberna@ua.es; Tel.: 34-965903533; Fax: 34-965903667.

${ }^{\ddagger}$ Dep. of Physics and Mathematics, UDLAP, 72820 San Andrés Cholula, Puebla, Mexico. E-Mail: francisco.guerra@udlap.mx.

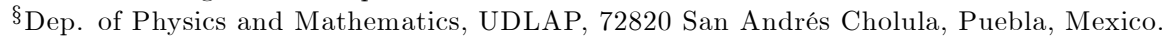
On leave from IMI-BAS, Sofia, Bulgaria. E-Mail: maxim.todorov@udlap.mx. 
We assume that the feasible set of $P$, denoted by $X$, is non-empty. Obviously, $X$ is a closed convex set whereas its image by the objective function $f(X) \subset \mathbb{R}^{p}$ (the objective space) is convex but possibly non-closed (unless $X$ is a bounded set or a polyhedral convex set). The VSIO problem $P$ can be reformulated as a VO problem with the single concave constraint function $\varphi(x):=\min _{t \in T}\left(a_{t}^{\prime} x-b_{t}\right)$, called marginal function:

$$
P: " \min " f(x)=\left(c_{1}^{\prime} x, \ldots, c_{p}^{\prime} x\right) \text { s.t. } \varphi(x) \geq 0 .
$$

Throughout the paper we use the following notation. Given $x, y \in \mathbb{R}^{m}$, we write $x \leqq y(x<y)$ when $x_{i} \leq y_{i}\left(x_{i}<y_{i}\right.$, respectively $)$ for all $i=1, \ldots, m$. Moreover, we write $x \leq y$ when $x \leqq y$ and $x \neq y$.

An element $x \in X$ is said to be efficient (weakly efficient) if there is no $\widehat{x} \in X$ such that $f(\widehat{x}) \leq f(x)(f(\widehat{x})<f(x)$, respectively). Analogously, $x \in X$ is said to be strongly efficient if there is no $\widehat{x} \in X \backslash\{x\}$ such that $f(\widehat{x}) \leqq f(x)$. There are many notions of proper efficiency in the literature, as those introduced by Geoffrion, Benson, Borwein and Henig. Since $P$ is convex, all these concepts are equivalent (see, e.g., [8]), so that we recall only Geoffrion's definition: a feasible point $x \in X$ is said to be properly efficient if there exists $\rho>0$ such that, for all $i=1, \ldots, p$ and $\widehat{x} \in X$ satisfying $c_{i}^{\prime} \widehat{x}<c_{i}^{\prime} x$, there exists $j \in\{1, \ldots, p\}$ such that $c_{j}^{\prime} \widehat{x}>c_{j}^{\prime} x$ and $\frac{c_{i}^{\prime} x-c_{i}^{\prime} \widehat{x}}{c_{j}^{\prime} \widehat{x}-c_{j}^{\prime} x} \leq \rho$. Another concept of proper efficiency in continuous linear VSIO involving directional derivatives of $\varphi$ (hardly computable in the general case) will be introduced later.

We denote by $X_{p E}, X_{s E}, X_{E}$, and $X_{w E}$ the sets of properly efficient points, strongly efficient points, efficient points, and weakly efficient points of $P$, respectively. Obviously, $X_{p E} \cup X_{s E} \subset X_{E} \subset X_{w E}$, with $X=X_{w E}$ whenever one component of $f$ is identically zero, and $X=X_{p E}$ in the trivial case that $f$ is the null function. Moreover, it is known that $f\left(X_{p E}\right)$ is dense in $f\left(X_{E}\right)$ ([16]; see also [8, Theorem 3.17]), so that $X_{p E}$ is dense in $X_{E}$ whenever $\left\{c_{1}, \ldots, c_{p}\right\}$ is linearly independent and either $p=n$ or $X_{p E}$ is bounded.

Given a (possibly nonlinear) VSIO problem

$$
P: " \min " f(x) \text { s.t. } x \in X \text {, }
$$

$x \in X$ is said to be locally (properly, strongly, weakly) efficient solution of $P$ if there exists $\varepsilon>0$ such that $x$ is (properly, strongly, weakly) efficient solution of

$$
P_{\varepsilon}: " \min " f(x) \text { s.t. } x \in X \cap B(\bar{x}, \varepsilon) .
$$

Global and local concepts coincide in linear VSIO thanks to the convexity of $X$ and the linearity of $f$. For instance, if $x \in X$ is not weakly efficient there exists $\widehat{x} \in X$ such that $f(\widehat{x})<f(x)$; since $f(\lambda \widehat{x}+(1-\lambda) x)<f(x)$ for all $\lambda \in] 0,1[$, with $\lambda \widehat{x}+(1-\lambda) x \in B(x, \varepsilon) \cap X$ for $\lambda$ sufficiently small, $x$ cannot be a locally weakly efficient solution of $P$. For this reason, in linear VSIO, we can characterize the (proper, strong, weak) efficiency on the basis of local information.

The known tests for nonlinear VO classify a given $x \in X$ as locally (properly, strongly, weakly) efficient solution or not through conditions involving subsets of 
the objective space $\mathbb{R}^{p}$ or suitable scalarizations of $P$ (see, e.g., [3], [8]). In this paper, on linear VSIO, we give Karush-Kuhn-Tucker (KKT in short) conditions for $x \in X_{p E}, x \in X_{s E}, x \in X_{E}$, and $x \in X_{w E}$ which are based on geometric tests involving subsets of the decision space or the space of coefficients $\left(\mathbb{R}^{n}\right.$ or $\mathbb{R}^{n+1}$ ) which can be computed from $x$ and the data describing $P$. Three convex cones related with the data in $P$ are basic in our approach: the convex conical hull of the gradients of the components of $f$,

$$
G:=\text { cone }\left\{c_{1}, \ldots, c_{p}\right\},
$$

that we shall call gradient cone, the characteristic cone,

$$
K:=\text { cone }\left\{\left(a_{t}, b_{t}\right), t \in T ;\left(0_{n},-1\right)\right\},
$$

and the active cone at $x \in X$,

$$
A(x):=\text { cone }\left\{a_{t}, t \in T(x)\right\},
$$

where $T(x):=\left\{t \in T: a_{t}^{\prime} x=b_{t}\right\}$ is the set of active indices at $x$, with $A(x)=$ $\left\{0_{n}\right\}$ whenever $T(x)=\emptyset$. Observe that computing $T(x)$ consists of finding all zeros of the mapping $t \mapsto a_{t}^{\prime} x-b_{t}$ on $T$, an easy problem whenever $T$ is a one-dimensional interval and the coefficient functions are polynomial in the variable $t$ (if, alternatively, these functions are analytic, $T(x)$ is finite whenever $T(x) \neq T)$.

The characteristic and active cones provide duality and optimality theorems in linear SIO. In particular, a given $x \in X$ is optimal if and only if the (one dimensional) gradient cone $G$ lies in the closure of $A(x)$, so that $G \subset A(x)$ is sufficient for the optimality of $x$, and it is also necessary when the constraints satisfy certain property called constraint qualification (c.q. in short). A c.q. is called global when it involves the whole set of constraints and local when it refers to those constraints which are active at $x$. Different c.q. for scalar nonconvex infinite and semi-infinite optimization problems are discussed in [24]. In linear VSIO, the necessary conditions for $x$ to be (properly, strongly, weakly) efficient are also expressed, under suitable c.q., in terms of $G$ and $A(x)$. Although linear and nonlinear VSIO have been investigated since the last 1980s from different perspectives, e.g., the pseudo-Lipschitz property and the lower and upper semicontinuity of the efficient set mapping under different types of perturbations, and its well-posedness ([5], [6], [7], [9], [17], [30], [31], [32], [33]), to the authors knowledge, only [4] provides KKT conditions for $x \in X_{p E}, x \in X_{E}$ and $x \in X_{w E}$ for a class of VSIO problems involving differentiable functions whose constraints satisfy certain invex-type conditions and are required to depend continuously on an index $t$ ranging on some compact topological space $T$. In particular, no previous work deals with constraint qualifications in linear VSIO or describes potential applications of this optimization branch.

This paper is organized as follows. Section 2 introduces different tangent cones at a given $x \in X$. In Section 3 we introduce four local c.q. and two global c.q., which are used in linear SIO or extend well-known c.q. from scalar 
optimization. The global c.q. are illustrated with several examples in Section 4. Finally, Sections 5 and 6 provide tests for the (proper, strong, weak) efficiency of a given $x \in X$ involving the gradient cone $G$, the active cone at $x, A(x)$, the tangent cones at $x \in X$ introduced in Section 2, and the existence of KKT multipliers, for general and for continuous linear VSIO problems, respectively.

\section{Tangent cones}

We start this section introducing the necessary notation and concepts. Given $Z \subset \mathbb{R}^{n}$, int $Z, \operatorname{cl} Z$, and bd $Z$ denote the interior, the closure, and the boundary of $Z$, respectively. The scalar product of $x, y \in \mathbb{R}^{n}$ is denoted by $x^{\prime} y$, the Euclidean norm of $x$ by $\|x\|$, the canonical basis by $\left\{e_{1}, \ldots, e_{n}\right\}$, and the zero vector by $0_{n}$. Given a linear mapping $g: \mathbb{R}^{n} \rightarrow \mathbb{R}^{p}$, we denote by ker $g$ its kernel, i.e., $\operatorname{ker} g=g^{-1}\left(0_{p}\right)$.

Given $Z \subset \mathbb{R}^{n}$, we denote by $\operatorname{rint} Z, \operatorname{span} Z$, and $\operatorname{conv} Z$ the relative interior, the linear span, and the convex hull of $Z$, , respectively, while cone $Z:=$ $\mathbb{R}_{+}$conv $Z$ denotes the convex conical hull of $Z \cup\left\{0_{n}\right\}$. The orthogonal complement of a linear subspace $Z$ is $Z^{\perp}:=\left\{y \in \mathbb{R}^{n}: x^{\prime} y=0 \forall x \in Z\right\}$. If $Z$ is a convex cone, its positive (negative) polar cone is $Z^{+}:=\left\{y \in \mathbb{R}^{n}: x^{\prime} y \geq 0 \forall x \in Z\right\}$ $\left(Z^{-}:=\left\{y \in \mathbb{R}^{n}: x^{\prime} y \leq 0 \forall x \in Z\right\}\right.$, respectively).

Given a closed convex set $Z \subset \mathbb{R}^{n}$, we associate with each $x \in Z$ four "tangent" cones:

The cone of feasible directions at $x$ is

$$
D(Z ; x)=\left\{d \in \mathbb{R}^{n}: \exists \mu>0 \text { such that } x+\mu d \in Z\right\} .
$$

The attainable cone at $x$, denoted by $A(Z ; x)$, is formed by those $d \in \mathbb{R}^{n}$ such that there exist $\tau>0$ and a vector function $h \in \mathcal{C}^{1}\left([0, \tau), \mathbb{R}^{n}\right)$ with $h(0)=x$, $h^{\prime}(0)=d$, and $h(s) \in Z$ for all $s \in[0, \tau)$. The attainable cone $A(Z ; x)$ is closed according to [25, Theorem 10].

The Bouligand tangent cone at $x$, denoted by $T(Z ; x)$, is formed by those $d \in \mathbb{R}^{n}$ such that there exist sequences $\left\{s^{k}\right\}_{k \in \mathbb{N}}$ and $\left\{d^{k}\right\}_{k \in \mathbb{N}}$ such that $s^{k} \rightarrow 0^{+}$, $d^{k} \rightarrow d$ as $k \rightarrow \infty$ and $x+s^{k} d^{k} \in Z$ for all $k \in \mathbb{N}$. In that case, since $d^{k} \in D(Z ; x)$ for all $k \in \mathbb{N}, d \in \operatorname{cl} D(Z ; x)$.

The interior tangent cone at $x$, denoted by $T^{i}(Z ; x)$, is formed by those $d \in \mathbb{R}^{n}$ such that there exist $\tau>0$ and a neighborhood $\mathcal{N}$ of $d$ such that $x+s \mathcal{N} \subset Z$ for all $s \in] 0, \tau[$.

Obviously,

$$
T^{i}(Z ; x) \subset D(Z ; x) \subset A(Z ; x) \subset T(Z ; x),
$$

all these cones being convex. It is known that $T^{i}(Z ; x)=\operatorname{int} D(Z ; x)$. Moreover, since $D(Z ; x) \subset A(Z ; x) \subset T(Z ; x) \subset \operatorname{cl} D(Z ; x)$ and $T(Z, x)$ is closed (cf. e.g. $[20])$, we have

$$
\operatorname{cl} D(Z ; x)=A(Z ; x)=T(Z ; x) .
$$

The next lemma will be used later. 
Lemma 1 ([28]) Let $Z_{1}$ and $Z_{2}$ be closed convex subsets of $\mathbb{R}^{n}$ and $x \in Z_{1} \subset$ $Z_{2}$. Then, $T^{i}\left(Z_{1} ; x\right) \subset T^{i}\left(Z_{2} ; x\right)$ and $T\left(Z_{1} ; x\right) \subset T\left(Z_{2} ; x\right)$.

Example 2 Consider the linear VSIP problem

$$
P: " \min " f(x)=\left(x_{1}, x_{2}\right) \text { s.t. } t x_{1}+(1-t) x_{2} \geq t-t^{2}, t \in[0,1],
$$

and the point $\bar{x}=(1,0)$. Since the feasible set of $P$ is $X=\left\{x \in \mathbb{R}_{+}^{2}: \sqrt{x_{1}}+\sqrt{x_{2}}=1\right\}+$ $\mathbb{R}_{+}^{2}$, (see [12, Example 1.1]), $T^{i}(X ; \bar{x})=\mathbb{R} \times \mathbb{R}_{++}, D(X ; \bar{x})=\left(\mathbb{R} \times \mathbb{R}_{++}\right) \cup$ cone $\{(1,0)\}$, so that the first two inclusions in (2) are strict while $A(X ; \bar{x})=$ $T(X ; \bar{x})=\mathbb{R} \times \mathbb{R}_{+}$.

\section{Constraint qualifications}

Next we introduce six constraint qualifications which are frequently encountered in the linear SIO literature or are inspired in classical constraint qualifications of nonlinear optimization.

\subsection{The Farkas-Minkowski c.q.}

The homogeneous Farkas Lemma ([12, Corollary 3.1.3]) establishes that $a^{\prime} x \geq 0$ is consequence of $\left\{a_{t}^{\prime} x \geq 0, t \in T\right\}$ (i.e., $a^{\prime} x \geq 0$ for all $t \in T$ implies that $\left.a^{\prime} x \geq 0\right)$ if and only if $a \in \operatorname{cl}$ cone $\left\{a_{t}, t \in T\right\}$, i.e.,

$$
\left\{\begin{array}{l}
a_{t}^{\prime} x \geq 0, t \in T \\
a^{\prime} x<0
\end{array}\right\} \text { is consistent } \Leftrightarrow a \notin \operatorname{cl} \text { cone }\left\{a_{t}, t \in T\right\} .
$$

while its non-homogeneous version ([12, Theorem 3.1]) asserts that, under the assumption that $X$ (the feasible set of $P$ ) is non-empty, $a^{\prime} x \geq b$ is consequence of $\left\{a_{t}^{\prime} x \geq b_{t}, t \in T\right\}$ if and only if $(a, b) \in \operatorname{cl} K$.

We say that $P$ satisfies the Farkas-Minkowski c.q. (FMCQ) when any consequence of $\left\{a_{t}^{\prime} x \geq b_{t}, t \in T\right\}$ is also consequence of some finite subsystem. Since the characteristic cone of a finite system is always closed, FMCQ holds if and only if $K$ is closed. Thus FMCQ is a (global) closed cone c.q.

The possible failure of FMCQ is not due to the geometry of the closed convex set $X$ but to its deficient linear representation. Indeed, let

$$
S=\{(a, b) \in \operatorname{cl} K:\|(a, b)\|=1\}
$$

and

$$
P_{1}: " \min " f(x)=\left(c_{1}^{\prime} x, \ldots, c_{p}^{\prime} x\right) \text { s.t. } a^{\prime} x \geq b,(a, b) \in S .
$$

Then, the feasible set of $P_{1}$ is $X_{1}=X$ (by the separation theorem) but $P_{1}$ satisfies FMCQ because its characteristic cone, cone $S=\operatorname{cl} K$, is closed. 


\subsection{The local Farkas-Minkowski c.q.}

We say that $P$ satisfies the local Farkas-Minkowski c.q. (LFMCQ) at $\bar{x} \in X$ when any consequence of $\left\{a_{t}^{\prime} x \geq b_{t}, t \in T\right\}$ determining a supporting hyperplane to $X$ at $\bar{x}$ is also consequence of some finite subsystem. Obviously, If $P$ satisfies FMCQ, then it satisfies LFMCQ at any $\bar{x} \in X$. The next example shows that the converse statement is not true.

Example 3 The system $\left\{x_{1}+t^{2} x_{2} \geq 2 t, t \in \mathbb{R}_{++}\right\}$is a linear representation of $X=\left\{x \in \mathbb{R}^{2}: x_{1} \geq 0, x_{1} x_{2} \geq 1\right\}$ (bd $X$ is a branch of hyperbola and $x_{1}+$ $t^{2} x_{2}=2 t$ is the tangent line to $\operatorname{bd} X$ at $\left.\left(t, \frac{1}{t}\right)\right)$. Then any consequence of the system determining a supporting hyperplane is the consequence of exactly one constraint. Nevertheless, there are consequences of the system, like $x_{1} \geq 0$ and $x_{2} \geq 0$, which are not consequence of a finite subsystem. Thus LFMCQ holds at any $\bar{x} \in X$ while FMCQ fails.

This c.q. can be characterized in terms of the relationship between the convex cones $A(\bar{x})$ and $D(X ; \bar{x})^{+}$, for which it is always true the inclusion

$$
A(\bar{x}) \subset D(X ; \bar{x})^{+} .
$$

In fact, according to [12, Theorem 5.7], $P$ satisfies LFMCQ at $\bar{x} \in X$ if and only if $A(\bar{x})=D(X ; \bar{x})^{+}$. So, the closedness of the active cone is a necessary condition for the LFMCQ.

\subsection{The local polyhedral c.q.}

Given $\bar{x} \in X$, taking polars in both members of (5) we get $D(X ; \bar{x}) \subset \operatorname{cl} D(X ; \bar{x}) \subset$ $A(\bar{x})^{+}$. The inclusion $D(X ; \bar{x}) \subset A(\bar{x})^{+}$is usually strict as $D(X ; \bar{x})$ is not necessarily closed. The equation $D(X ; \bar{x})=A(\bar{x})^{+}$holds when $T$ is finite, in which case $X$ is a polyhedral convex set.

We say that $P$ satisfies the local polyhedral c.q. (LOPCQ) at $\bar{x} \in X$ when $D(X ; \bar{x})=A(\bar{x})^{+}$. In that case, $D(X ; \bar{x})$ is closed. When $P$ satisfies LOPCQ at any $\bar{x} \in X$, then $X$ is a quasipolyhedral set, i.e., the non-empty intersections of $X$ with polytopes are polytopes. If $P$ satisfies LOPCQ at $\bar{x} \in X$, then it satisfies LFMCQ at $\bar{x}$ too ([12, Theorem 5.6 ]). Example 3 shows that the converse statement does not hold (there LFMCQ holds at any $x \in X$ but the intersection of $X$ with a polytopes is not necessarily a polytope).

\subsection{The Slater c.q.}

We say that $P$ satisfies the Slater constraint qualification (SCQ) if there exists an element $\widehat{x}$ (called Slater point) such that $T(\widehat{x})=\emptyset$, i.e., $a_{t}^{\prime} \widehat{x}>b_{t}$ for all $t \in T$.

When $T$ is finite, SCQ holds if and only if int $X \neq \emptyset$ and no constraint is trivial $\left(0_{n}^{\prime} x \geq 0\right)$, so that $\mathrm{SCQ}$ is an interior-type c.q. This global c.q. is only useful when $P$ is continuous. In fact, it is even possible that $A(x)=\left\{0_{n}\right\}$ for 
all $x \in X$ for a VSIO problem satisfying SCQ: it is enough to replace each constraint $a_{t}^{\prime} x \geq b_{t}$ of $P$ by the system $\left\{a_{t}^{\prime} x \geq b_{t}-\frac{1}{k}, k \in \mathbb{N}\right\}$, so that all constraints are inactive at any $x \in X$ for the new linear representation of $X$. When $P$ is continuous and satisfies SCQ, then $\left\{\left(a_{t}, b_{t}\right), t \in T\right\}$ is a compact subset of $\mathbb{R}^{n+1}$ whose elements form an acute angle with $(\widehat{x},-1)$, so that the convex cone generated by conv $\left\{\left(a_{t}, b_{t}\right), t \in T ;\left(0_{n},-1\right)\right\}$ (i.e., the characteristic cone $K$ ) is closed. Thus, FMCQ holds. In that case, int $X$ coincides with the set of Slater points (by [12, Theorem 5.9]). Consequently, SCQ implies int $X \neq \emptyset$, and the converse hods whenever $a_{t} \neq 0_{n}$ for all $t \in T$.

\subsection{The local Slater c.q.}

If $\widehat{x} \in X$ is a Slater point and $t \in T(\bar{x})$, then $a_{t}^{\prime}(\widehat{x}-\bar{x})=a_{t}^{\prime} \widehat{x}-b_{t}>0$. The inequality $a_{t}^{\prime} d>0$ for $d:=\widehat{x}-\bar{x}$ suggest another local c.q. which will be satisfied at any feasible point whenever SCQ holds.

We say that $P$ satisfies the local Slater constraint qualification (LSCQ) at $\bar{x} \in X$ (or the Mangasarian-Fromovitz c.q. at $\bar{x} \in X$ ) when either $T(\bar{x})=\emptyset$ or there exists a vector $d \in \mathbb{R}^{n}$ satisfying

$$
a_{t}^{\prime} d>0 \text { for all } t \in T(\bar{x}) .
$$

If LSCQ holds at $\bar{x} \in X$ and $T \backslash T(\bar{x})$ is finite, taking $d$ as in (6), we have $a_{t}^{\prime}(\bar{x}+\lambda d)>b_{t}$ for all $t \in T(\bar{x})$ and $\lambda>0$, by (6), while $a_{t}^{\prime}(\bar{x}+\lambda d)>b_{t}$ for all $t \in T \backslash T(\bar{x})$ and $\lambda>0$ sufficiently small, so that SCQ holds. In particular, when $T$ is finite, SCQ holds if and only if LSCQ holds at some $\bar{x} \in X$. The next example shows that the finiteness assumption in the latter statement cannot be replaced by the weaker one that $P$ is continuous.

Example 4 Consider the linear system

$$
\left\{(1-t) x_{1}+t x_{2} \geq 0, t \in[0,1] ;-x_{1}-x_{2} \geq 2-t, t \in[2,3]\right\},
$$

whose characteristic cone is $K=\mathbb{R}^{2} \times \mathbb{R}_{-}$. For $\bar{x}=0_{2}$ (the unique element of $X$ ) and $d=(1,1)$ we have $a_{t}^{\prime} d=1>0$ for all $t \in T(\bar{x})=[0,1]$, so that $L S C Q$ holds at $\bar{x}$ despite of the failure of $S C Q$.

Lemma 5 If $P$ is continuous and satisfies $L S C Q$ at $\bar{x} \in X$, then $A(\bar{x})$ is closed.

Proof: We can assume $T(\bar{x}) \neq \emptyset$ (otherwise $A(\bar{x})=\left\{0_{n}\right\}$ is closed). The continuity assumption implies that $T(\bar{x})$ is a compact set, as well as $\left\{a_{t}, t \in T(\bar{x})\right\}$. By $(6)$, conv $\left\{a_{t}, t \in T(\bar{x})\right\}$ is a compact convex set which does not contain the origin. So $A(\bar{x})=$ cone $\left\{a_{t}, t \in T(\bar{x})\right\}$ is closed.

The following example shows that, given a continuous linear VSIO problem $P, A(\bar{x})$ may be closed although it does not satisfy LSCQ at $\bar{x}$.

Example 6 Consider the linear VSIO problem

$P: " \min " f(x)=\left(x_{1}, x_{2}\right)$ s.t. $-t x_{1}-x_{2} \geq 0, t \in[0,1] ;-t x_{1}+x_{2} \geq 0, t \in[0,1]$ 
and $0_{2} \in X=\left\{x \in \mathbb{R}^{2}: x_{1} \leq 0, x_{2}=0\right\}$ (a one-dimensional cone). Since all the constraints are active at $0_{2}$,

$$
A\left(0_{2}\right)=\text { cone }\{(-t, \pm 1): 0 \leq t \leq 1\}=\mathbb{R}_{-} \times \mathbb{R},
$$

which is closed. However, since the two vectors $a_{t}$ corresponding to $t=0$ are opposite to each other, (6) has no solution. That is, $L S C Q$ is not fulfilled at $0_{2}$. Observe that $A\left(0_{2}\right)^{+}=X=D\left(X, 0_{2}\right)$, so that $L O P C Q$ and $L F M C Q$ hold at $0_{2}$.

\subsection{The extended Kuhn-Tucker constraint qualification}

The next c.q. was introduced in [29] for infinite programming problems as an extension of that used by H. W. Kuhn and A. W. Tucker in [19] for the finite case. Its definition involves the directional derivative of $\varphi$ at $\bar{x} \in X$ in a direction $d \in \mathbb{R}^{n}$, denoted by $\varphi^{\prime}(\bar{x} ; d)$.

We say that $P$ satisfies the extended Kuhn-Tucker c.q. (EKTCQ) at $\bar{x} \in X$ when

$$
\left\{d \in \mathbb{R}^{n}: \varphi^{\prime}(\bar{x} ; d) \geq 0\right\} \subset A(X ; \bar{x}) .
$$

As a consequence of the Ioffe and Tihkomirov's theorem on the subdifferential of the supremum function (see e.g. [34, Theorem 2.4.18] and [15, Proposition $6.3]$ ), when $P$ is continuous and $\bar{x} \in \operatorname{bd} X$, then

$$
\varphi^{\prime}(\bar{x} ; d)=\min _{t \in T(\bar{x})} a_{t}^{\prime} d .
$$

(more general formulas for $\varphi^{\prime}(\bar{x} ; d)$ can be derived from those corresponding to the subdifferential of the supremum function in [15, Proposition 4.1] and [22, Theorem 1], but they are too complex for this framework). It is worth observing that $\varphi$ is directionally differentiable at $\bar{x}$ in the Hadamard sense (cf. e.g. [2, Proposition 2.49]), i.e.,

$$
\varphi^{\prime}(\bar{x} ; d)=\lim _{\substack{s \rightarrow 0^{+} \\ d^{\prime} \rightarrow d}} \frac{\varphi\left(\bar{x}+s d^{\prime}\right)-\varphi(\bar{x})}{s} .
$$

Consider again the set $X$ of Example 2. Obviously, FMCQ and SCQ hold, so that LFMCQ and LSCQ also hold at any $x \in X$. In the contrary, LOPCQ fails

at $\bar{x}=(1,0)$ because $D(X ; \bar{x})$ is not even closed. In order to check the EKTCQ at $\bar{x}$ we must identify the function $\varphi^{\prime}(\bar{x}, \cdot)$. Although the function $t \mapsto a_{t}^{\prime} x-b_{t}$ is quadratic for all $x$, the expression of $\varphi$,

$$
\begin{aligned}
\varphi(x) & =\min _{t \in[0,1]}\left(t x_{1}+(1-t) x_{2}-t+t^{2}\right) \\
& = \begin{cases}-\frac{\left(x_{1}-x_{2}\right)^{2}}{4}+\frac{x_{1}+x_{2}}{2}-\frac{1}{4}, & \text { if }\left|x_{1}-x_{2}\right| \leq 1 \\
\min \left\{x_{1}, x_{2}\right\}, & \text { otherwise, }\end{cases}
\end{aligned}
$$

is too involved to compute $\varphi^{\prime}(\bar{x}, \cdot)$ directly from the definition or from (9). So, it is preferable to apply (8), with $T(\bar{x})=\{0\}$, to get

$$
\varphi^{\prime}(\bar{x} ; d)=\min _{t \in T(\bar{x})}\left(t d_{1}+(1-t) d_{2}\right)=d_{2} .
$$


Since

$$
\left\{d \in \mathbb{R}^{n}: \varphi^{\prime}(\bar{x} ; d) \geq 0\right\}=\mathbb{R} \times \mathbb{R}_{+}=A(X ; \bar{x}),
$$

we conclude that EKTCQ also holds at $\bar{x}$.

The next lemma provides useful approximations of the tangent cones to $X$ at $\bar{x} \in$ bd $X$ in terms of the directional derivative $\varphi^{\prime}(\bar{x} ; d)$.

Lemma 7 ([14, Lemma 3.2]) Let $P$ be continuous and $\bar{x} \in \operatorname{bd} X$. Then,

$$
\left\{d \in \mathbb{R}^{n}: \varphi^{\prime}(\bar{x}, d)>0\right\} \subset T^{i}(X ; \bar{x}) \subset T(X ; \bar{x}) \subset\left\{d \in \mathbb{R}^{n}: \varphi^{\prime}(\bar{x}, d) \geq 0\right\} .
$$

Remark 8 If $T(\bar{x}) \neq \emptyset$ and $d$ is as in (7), then the vector function $h(s)=\bar{x}+s d$ satisfies

$$
\begin{aligned}
h(0) & =\bar{x} \\
h^{\prime}(0) & =d, \\
a_{t}^{\prime} h(s) & \geq b_{t}, \text { for all } s \geq 0 \text { and } t \in T(\bar{x}) .
\end{aligned}
$$

Moreover, for each $t \in T \backslash T(\bar{x})$, there exists $\tau(t)>0$ such that

$$
a_{t}^{\prime} h(s) \geq b_{t}, \text { for all } s \in[0, \tau(t))[.
$$

If $T \backslash T(\bar{x})$ is finite, then we can take $\tau=\inf _{t}\{\tau(t)\}>0$ and EKTCQ is automatically satisfied. Otherwise, it may be that $\inf _{t}\{\tau(t)\}=0$ and EKTCQ may not hold.

Lemma 9 If $P$ is continuous and satisfies $L S C Q$ at $\bar{x} \in \operatorname{bd} X$, then $P$ satisfies EKTCQ at $\bar{x}$ too.

Proof: Since LSCQ is satisfied at $\bar{x}$ and $T(\bar{x})$ is compact, (8) implies that $\left\{d \in \mathbb{R}^{n}: \varphi^{\prime}(\bar{x} ; d)>0\right\} \neq \emptyset$ and

$$
\operatorname{cl}\left\{d \in \mathbb{R}^{n}: \varphi^{\prime}(\bar{x} ; d)>0\right\}=\left\{d \in \mathbb{R}^{n}: \varphi^{\prime}(\bar{x} ; d) \geq 0\right\} .
$$

So, by Lemma $7,\left\{d \in \mathbb{R}^{n}: \varphi^{\prime}(\bar{x} ; d) \geq 0\right\}=T(X ; \bar{x})=A(X ; \bar{x})$.

The following example shows that the converse statement of Lemma 9 does not hold.

Example 10 Consider the system in Example 6 and $\bar{x}=0_{2} \in$ bd $X$. If $d \in \mathbb{R}^{2}$ satisfies $\varphi^{\prime}(\bar{x} ; d) \geq 0$, then $-t d_{1}-d_{2} \geq 0$ and $-t d_{1}+d_{2} \geq 0$ for all $t \in[0,1]$, i.e., $d \in X$ (a one-dimensional cone). Thus, the vector function $h(s):=\left(s d_{1}, s d_{2}\right)$ satisfies that $h(s) \in X$, for all $s \geq 0, h(0)=\bar{x}$ and $h^{\prime}(0)=d$. That is $d \in$ $A(X ; \bar{x})$.

Lemma 11 Let $P$ be continuous and $\bar{x} \in \mathrm{bd} X$. Then, $P$ satisfies EKTCQ at $\bar{x}$ if and only if

$$
T(X ; \bar{x})=\left\{d \in \mathbb{R}^{n}: \varphi^{\prime}(\bar{x}, d) \geq 0\right\} .
$$

Proof: Assume that $P$ satisfies EKTCQ at $\bar{x}$, i.e.,

$$
\left\{d \in \mathbb{R}^{n}: \varphi^{\prime}(\bar{x}, d) \geq 0\right\} \subset A(X ; \bar{x})=T(X ; \bar{x}) .
$$

By (11) and Lemma 7, (10) holds. The converse statement is trivial. 


\section{Global c.q. in selected applications}

In this section we show that linear VSIO problems arise in a natural way in different fields and analyze the fulfilment of the two global constraint qualifications introduced in the previous section: SCQ and FMCQ.

\subsection{Robust linear vector optimization}

Let

$$
P_{0}: \text { "min" } f(x)=\left(c_{1}^{\prime} x, \ldots, c_{p}^{\prime} x\right) \text { s.t. } a_{i}^{\prime} x \geq b_{i}, i=1, \ldots, q,
$$

be a linear VO problem with uncertain data.

Firstly, we assume that the uncertainty is confined to the cost vectors, i.e., the constraints remain fixed. Let $c_{i}$ range on certain set $C_{i} \subset \mathbb{R}^{n}, i=1, \ldots, p$. The robust perspective corresponds to a pessimistic decision maker, for which the cost vector of deciding $x \in \mathbb{R}^{n}$ such that $a_{i}^{\prime} x \geq b_{i}, i=1, \ldots, q$, will be worst possible, i.e., $\left(\max _{c_{1} \in C_{1}} c_{1}^{\prime} x, \ldots, \max _{c_{p} \in C_{p}} c_{p}^{\prime} x\right)$. Then, the robust counterpart of $P_{0}$ is the linear VSIO problem,

$$
\begin{aligned}
& P_{1}: \quad \text { "min" } f(x, z)=\left(z_{1}, \ldots, z_{p}\right) \\
& \text { s.t. } \quad z_{j} \geq c_{j}^{\prime} x, c_{j} \in C_{j}, j=1, \ldots, p \text {, } \\
& a_{i}^{\prime} x \geq b_{i}, i=1, \ldots, q,
\end{aligned}
$$

whose decision space is $\mathbb{R}^{n+p}$ (so, the initial problem is embedded into a higher dimensional decision space and the number of constraints turns out to be infinite). If $\left\{C_{j}, j=1, \ldots, p\right\}$, is a family of compact sets, then $P_{1}$ is continuous and SCQ holds if and only if the there exists $\widehat{x}$ such that $a_{i}^{\prime} \widehat{x}>b_{i}, i=1, \ldots, q$, in which case FMCQ holds too.

Secondly, let us assume that the uncertain data in $P_{0}$ are the coefficient vectors $\left(a_{i}, b_{i}\right), i=1, \ldots, q$. Let us assume that each vector $\left(a_{i}, b_{i}\right)$ ranges on a given set $S_{i} \subset \mathbb{R}^{n+1}, i=1, \ldots, q$, whereas $c$ remains fixed. The robust approach tries to guarantee the feasibility of the selected decision under any conceivable circumstance. Thus, we must solve the linear VSIO problem

$$
P_{2}: " \min " \quad f(x)=\left(c_{1}^{\prime} x, \ldots, c_{p}^{\prime} x\right) \text { s.t. } a^{\prime} x \geq b,(a, b) \in \bigcup_{i=1}^{q} S_{i},
$$

whose decision space coincides with the initial one. If $S_{1}, \ldots, S_{q}$ are compact, then $P_{2}$ is continuous and satisfies FMCQ whenever cone $\left(\bigcup_{i=1}^{q} S_{i} \cup\left\{\left(0_{n},-1\right)\right\}\right)$ is closed.

Finally, we assume that all the data in $P_{0}$ are uncertain. Combining the previous arguments we get the following robust counterpart of $P_{0}$ :

$$
\begin{array}{cl}
P_{3}: \quad \text { "min" } & f(x, z)=\left(z_{1}, \ldots, z_{p}\right) \\
\text { s.t. } & z_{j} \geq c_{j}^{\prime} x, c_{j} \in C_{j}, j=1, \ldots, p, \\
& a^{\prime} x \geq b,(a, b) \in \bigcup_{i=1}^{q} S_{i} .
\end{array}
$$


If the sets $C_{1}, \ldots, C_{p}, S_{1}, \ldots, S_{q}$ are compact, then $P_{3}$ is continuous and satisfies SCQ if and only if $P_{2}$ satisfies it.

\subsection{Convex vector optimization}

Consider the vector optimization problem

$$
P_{0}: " \min " ~ h(x)=\left(h_{1}(x), \ldots, h_{p}(x)\right) \text { s.t. } g_{t}(x) \leq 0, t \in T \text {, }
$$

where all the involved functions are proper, lower semicontinuous, and convex from $\mathbb{R}^{n}$ to $\mathbb{R} \cup\{+\infty\}$, whereas the cardinality of $T$ is irrelevant. It is possible to reformulate $P_{0}$ as a linear VSIO problem by means of the Fenchel conjugates of the involved functions.

The effective domain of $f: \mathbb{R}^{n} \rightarrow \mathbb{R} \cup\{ \pm \infty\}$ is $\operatorname{dom} f=\left\{x \in \mathbb{R}^{n}: f(x)<\right.$ $+\infty\}$. The conjugate function of $f, f^{*}: \mathbb{R}^{n} \rightarrow \mathbb{R} \cup\{ \pm \infty\}$, is defined by

$$
f^{*}(v)=\sup \{\langle v, x\rangle-f(x): x \in \operatorname{dom} f\} .
$$

It is well-known that, if $f$ is a proper lower semicontinuous convex function, then $f^{*}$ enjoys the same properties and its conjugate, denoted by $f^{* *}: \mathbb{R}^{n} \rightarrow$ $\mathbb{R} \cup\{ \pm \infty\}$ and defined by

$$
f^{* *}(x)=\sup \left\{\langle v, x\rangle-f^{*}(v): v \in \operatorname{dom} f^{*}\right\},
$$

coincides with $f$.

From the assumptions on $P_{0}$ we get two consequences:

1st, each objective function is the supremum of affine functions because

$$
h_{i}(x)=h_{i}^{* *}(x)=\sup _{u \in \operatorname{dom} h_{i}^{*}}\left\{x^{\prime} u-h_{i}^{*}(u)\right\}, i=1, \ldots, p .
$$

2nd, since all the constraints in $P_{0}$ can be expressed as $g_{t}(x) \leq 0$, and $g_{t}^{* *}=g_{t}$, we have

$$
\begin{aligned}
& g_{t}(x) \leq 0 \Longleftrightarrow g_{t}^{* *}(x) \leq 0 \\
& \Longleftrightarrow x^{\prime} u-g_{t}^{*}(u) \leq 0 \forall u \in \operatorname{dom} g_{t}^{*} \\
& \Longleftrightarrow u^{\prime} x \leq g_{t}^{*}(u) \forall u \in \operatorname{dom} g_{t}^{*} .
\end{aligned}
$$

We associate with $P_{0}$ the linear VSIO problem

$$
\begin{array}{ccl}
P_{1}: & \text { "min" } & f(z, x)=\left(z_{1}, \ldots, z_{p}\right) \\
\text { s.t. } & -z_{i}+u^{\prime} x \leq h_{i}^{*}(u), u \in \operatorname{dom} h_{i}^{*}, i=1, \ldots, p, \\
& u^{\prime} x \leq g_{t}^{*}(u),(u, t) \in \operatorname{dom} g_{t}^{*} \times T,
\end{array}
$$

whose decision space is $\mathbb{R}^{n+p}$.

For $k=0,1$, we denote by $X^{k}\left(X_{E}^{k}, X_{s E}^{k}\right.$, and $\left.X_{w E}^{k}\right)$ the feasible set of $P_{k}$ (the sets of efficient points, strongly efficient points, and weakly efficient points of $P_{k}$, respectively). Given $x \in X^{0},-h_{i}(x)+u^{\prime} x \leq h_{i}^{*}(u)$, for all $u \in \operatorname{dom} h_{i}^{*}$, 
$i=1, \ldots, p$, and $u^{\prime} x \leq g_{t}^{*}(u)$, for all $(u, t) \in \operatorname{dom} g_{t}^{*} \times T$, by (12) and (13), respectively. So,

$$
x \in X^{0} \Leftrightarrow\left(x, h_{1}(x), \ldots, h_{p}(x)\right) \in X^{1} .
$$

Assume that $\left(x, h_{1}(x), \ldots, h_{p}(x)\right) \notin X_{E}^{1}$. Let $\left(\widehat{x}, \widehat{z}_{1}, \ldots, \widehat{z}_{1}\right) \in X^{1}$ be such that $\widehat{z}_{i} \leq h_{i}(x), i=1, \ldots, p$. Then $\widehat{x} \in X^{0}$ and

$$
h_{i}(\widehat{x})=\sup _{u \in \operatorname{dom} h_{i}^{*}}\left\{\widehat{x}^{\prime} u-h_{i}^{*}(u)\right\} \leq \widehat{z}_{i} \leq h_{i}(x), i=1, \ldots, p,
$$

so that $x \notin X_{E}^{0}$. So,

$$
x \in X_{E}^{0} \Leftrightarrow\left(x, h_{1}(x), \ldots, h_{p}(x)\right) \in X_{E}^{1} .
$$

Similar arguments show that

$$
x \in X_{s E}^{0} \Leftrightarrow\left(x, h_{1}(x), \ldots, h_{p}(x)\right) \in X_{s E}^{1}
$$

and

$$
x \in X_{w E}^{0} \Leftrightarrow\left(x, h_{1}(x), \ldots, h_{p}(x)\right) \in X_{w E}^{1} .
$$

In summary, the vector optimization problems $P_{0}$ and $P_{1}$ are equivalent in the sense that checking the efficiency (strong efficiency, weak efficiency) of the feasible points of $P_{0}$ can be reduced to an analogous question regarding associated feasible points of $P_{1}$.

We cannot expect the continuity of $P_{1}$ because $\operatorname{dom} h_{t}^{*}$ is usually an unbounded set (e.g., it is a singleton whenever $h_{t}$ is an affine function). The FMCQ is fulfilled by $P_{1}$ if and only if

$$
\begin{aligned}
-K_{1} & =\text { cone }\left\{\bigcup_{i=1}^{p}\left(\left\{-e_{i}\right\} \times \text { epi } h_{i}^{*}\right)\right\} \\
& + \text { cone }\left\{\bigcup_{t \in T}\left(\left\{0_{p}\right\} \times \operatorname{epi} g_{t}^{*}\right)\right\} \\
& + \text { cone }\left\{\left(0_{p+n}, 1\right)\right\}
\end{aligned}
$$

is closed.

\subsection{Portfolio management}

We can invest a capital $M$ in $n$ shares. For $i \in\{1, \ldots, n\}$, we denote by $x_{i}$ the amount to be invested in the $i$-th share, and by $r_{i}$ its rate of return. Obvious constraints are $\sum_{i=1}^{n} x_{i}=M$ and $x_{i} \geq 0, i=1, \ldots, n$. We express these, and possibly other linear constraints, in a compact way as $a_{i}^{\prime} x \geq b_{i}, i=1, \ldots, q$. In the (unrealistic) absence of uncertainty, the problem to be solved is the linear optimization (LO) one

$$
P_{0}: \max r^{\prime} x \text { s.t. } a_{i}^{\prime} x \geq b_{i}, i=1, \ldots, q .
$$


Unfortunately, $r$ is in practice an uncertain vector. The uncertain problem $P_{0}$ can be modeled in a variety of ways, taking into account that the decision maker intends to maximize its return at a minimum risk. If the probability distribution of $r$ is unknown, the first objective for a pessimistic decision maker consists of maximizing $\inf _{r \in R} r^{\prime} x$ (or, equivalently, minimizing $\sup _{r \in R}\left(-r^{\prime} x\right)$ ), where $R \subset \mathbb{R}^{n}$ denotes the set of conceivable values of $r$. Concerning the risk, it is usually identified with the variance of the portfolio $x$, i.e., the uncertain number $x^{\prime} V x$, where $V$ denotes the (positive definite) matrix of variances-covariances of $r$. So, the second objective consists of minimizing the quadratic convex function $h(x):=x^{\prime} V x$. Consequently, we have a biobjective convex optimization problem that can be reformulated as a linear VSIO problem as in the latter subsection. Indeed, since $h^{*}(u)=\frac{1}{4} u^{\prime} V^{-1} u$ for all $u \in \mathbb{R}^{n}$, the equivalent problem is

$$
\begin{array}{cl}
P_{1}: \quad \text { "min" } & f(z, x)=\left(z_{1}, z_{2}\right) \\
\text { s.t. } & r^{\prime} x+z_{1} \geq 0, r \in R, \\
& -u^{\prime} x+z_{2} \geq-\frac{1}{4} u^{\prime} V^{-1} u, u \in \mathbb{R}^{n}, \\
& a_{i}^{\prime} x \geq b_{i}, i=1, \ldots, q,
\end{array}
$$

whose decision space is $\left(z_{1}, z_{2}, x\right) \in \mathbb{R}^{n+2}$. Certainly, $P_{1}$ is not continuous, and it satisfies FMCQ if and only if its characteristic cone

$$
\begin{aligned}
K_{1} & =\text { cone }\{(1,0, r, 0), r \in R\} \\
& + \text { cone }\left\{\left(0,1,-u,-\frac{1}{4} u^{\prime} V^{-1} u\right), u \in \mathbb{R}^{n}\right\} \\
& + \text { cone }\left\{\left(0,0, a_{i}, b_{i}\right)\right\} \\
& + \text { cone }\left\{\left(0_{n+2},-1\right)\right\}
\end{aligned}
$$

is closed.

\subsection{Functional approximation}

Let $h, v_{1}, \ldots, v_{n}$ be Riemann integrable functions on $T=[\alpha, \beta], \alpha<\beta$. Consider the problem consisting of computing a "good" approximation to $h$ from above by means of linear combinations of $v_{1}, \ldots, v_{n}$, but it is not obvious how to measure the approximation error. If we choose the $L_{\infty}$ and the $L_{1}$ norms, the problem consists of the simultaneous minimization of the $L_{\infty}$ and the $L_{1}$ errors. If we consider a linear combination $\sum_{i=1}^{n} v_{i}(t) x_{i}$ such that $h(t) \leq \sum_{i=1}^{n} v_{i}(t) x_{i}$, for all $t \in T$ (a feasible decision of the approximation problem), the corresponding $L_{\infty}$ and the $L_{1}$ errors are

$$
\left\|h-\sum_{i=1}^{n} x_{i} v_{i}\right\|_{\infty}=\max _{t \in T}\left\{\sum_{i=1}^{n} x_{i} v_{i}(t)-h(t)\right\}
$$

and

$$
\begin{gathered}
\left\|h-\sum_{i=1}^{n} x_{i} v_{i}\right\|_{1}=\int_{\alpha}^{\beta}\left[\sum_{i=1}^{n} v_{i}(t) x_{i}-h(t)\right] d t \\
=\sum_{i=1}^{n}\left(\int_{\alpha}^{\beta} v_{i}(t) d t\right) x_{i}-\int_{\alpha}^{\beta} h(t) d t
\end{gathered}
$$


respectively. So, we have to solve

$$
\begin{array}{cl}
P_{1}: \text { "min" } & f\left(x, x_{n+1}\right)=\left(x_{n+1}, \sum_{i=1}^{n}\left(\int_{\alpha}^{\beta} v_{i}(t) d t\right) x_{i}\right) \\
\text { s.t. } & \sum_{i=1}^{n} v_{i}(t) x_{i} \geq h(t), t \in T, \\
& x_{n+1} \geq h(t)-\sum_{i=1}^{n} v_{i}(t) x_{i} \geq-x_{n+1}, t \in T .
\end{array}
$$

The approximating function $\sum_{i=1}^{n} v_{i}(t) x_{i}$ can be forced to satisfy conditions such as the requirement to be non-decreasing or convex on $T$, that can be expressed through the linear systems $\left\{\sum_{i=1}^{n} \frac{d v_{i}}{d t} x_{i} \geq 0, t \in T\right\}$ and $\left\{\sum_{i=1}^{n} \frac{d^{2} v_{i}}{d t^{2}} x_{i} \geq 0, t \in T\right\}$ (assuming that $v_{1}, \ldots, v_{n} \in \mathcal{C}^{1}(T)$ and $v_{1}, \ldots, v_{n} \in \mathcal{C}^{2}(T)$, respectively). In either case, $P_{1}$ is continuous if and only if $T$ is a compact Hausdorff topological space and $h, v_{1}, \ldots, v_{n} \in \mathcal{C}(T)$. Moreover, $P_{1}$ satisfies SCQ (and so FMCQ) if there exists some $\widehat{x}$ such that $\sum_{i=1}^{n} v_{i}(t) \widehat{x}_{i}>h(t)$ (together with $\sum_{i=1}^{n} \frac{d v_{i}}{d t} \widehat{x}_{i}>0$ and/or $\sum_{i=1}^{n} \frac{d^{2} v_{i}}{d t^{2}} \widehat{x}_{i}>0$ under the monotonicity and/or convexity requirement) for all $t \in T$.

\section{Cone and KKT conditions in general VSIO}

It is well-known (see, e.g. [8, Theorem 3.21 and Corollary 3.23]) that, if $T$ is finite, $0_{n} \notin \operatorname{conv}\left\{c_{1}, \ldots, c_{p}\right\}$ and $\bar{x} \in X, \bar{x}$ is a weakly efficient solution of the (ordinary) linear VO problem $P$ if and only if there exist $t^{j} \in T(\bar{x}), j=1, \ldots, q$, as well as nonnegative scalars $\lambda_{1}, \ldots, \lambda_{p}, \mu_{1}, \ldots, \mu_{q}$ satisfying

$$
\sum_{i=1}^{p} \lambda_{i} c_{i}=\sum_{j=1}^{q} \mu_{j} a_{t^{j}} \neq 0_{n} .
$$

In geometric terms, the KKT condition (14) asserts that $G \cap A(\bar{x}) \neq\left\{0_{n}\right\}$, both cones depending on the data (it is sufficient to take $q \leq n$ by Carathéodory's theorem applied to the convex cone $A(\bar{x})$ ). In this section we give similar conditions for linear VSIO problems and different types of efficiency.

Proposition 12 Given $\bar{x} \in X$, the following statements hold:

(i) $\bar{x} \in X_{s E}$ if and only if $D(X ; \bar{x}) \cap G^{-}=\left\{0_{n}\right\}$.

(ii) $\bar{x} \in X_{E}$ if and only if $D(X ; \bar{x}) \cap G^{-} \subset \operatorname{ker} f$.

(iii) If $0_{n} \in\left\{c_{1}, \ldots, c_{p}\right\}$, then, $X_{w E}=X$. Otherwise, $\bar{x} \in X_{w E}$ if and only if $D(X ; \bar{x}) \cap \operatorname{int} G^{-}=\emptyset$.

Proof. (i) Suppose that there exists $d \in D(X ; \bar{x}) \cap G^{-}, d \neq 0_{n}$. Let $\varepsilon>0$ be such that $\bar{x}+\varepsilon d \in X$. Taking $x:=\bar{x}+\varepsilon d \neq \bar{x}$, we have $f(x) \leqq f(\bar{x})$, so that $\bar{x} \notin X_{s E}$.

Conversely, if $\bar{x} \notin X_{s E}$ there exists $x \in X$ such that $x \neq \bar{x}$ and $f(x-\bar{x}) \leqq$ $0_{p}$, i.e., $c_{i}^{\prime}(x-\bar{x}) \leq 0, i=1, \ldots, p$. Denoting $d:=x-\bar{x}$, we have $d \neq 0_{n}$ and $d \in D(X ; \bar{x}) \cap G^{-}$, so that $D(X ; \bar{x}) \cap G^{-} \neq\left\{0_{n}\right\}$.

(ii) Suppose that there exists $d \in D(X ; \bar{x}) \cap G^{-}, d \notin \operatorname{ker} f$. From $d \in G^{-}$ and $d \notin \operatorname{ker} f$ we get $c_{i}^{\prime} d \leq 0, i=1, \ldots, p$, i.e., $f(d) \leqq 0_{p}$. Since $f(d) \neq 0_{p}$, we 
have $f(d) \leq 0_{p}$. From $d \in D(X ; \bar{x})$ there exists $\varepsilon>0$ such that $\bar{x}+\varepsilon d \in X$. Then,

$$
f(\bar{x}+\varepsilon d)=f(\bar{x})+\varepsilon f(d) \leq f(\bar{x})
$$

and so $\bar{x} \notin X_{E}$.

Now, suppose that $\bar{x}$ is not an efficient point, i.e., there exists $x \in X$ such that $f(x) \leq f(\bar{x})$. Obviously, $x-\bar{x} \in D(X ; \bar{x})$. Moreover, $f(x) \leq f(\bar{x})$ means that $c_{i}^{\prime}(x-\bar{x}) \leq 0, i=1, \ldots, p$, with at least one strict inequality, i.e., $x-\bar{x} \in G^{-}$ and $x-\bar{x} \notin \operatorname{ker} f$. Thus $D(X ; \bar{x}) \cap G^{-} \nsubseteq \mathbb{k e r} f$.

(iii) We have just to prove the second statement. Suppose that there exists $d \in D(X ; \bar{x}) \cap \operatorname{int} G^{-}$. Let $\delta>0$ be such that $c_{i}^{\prime}(d+\delta u) \leq 0$ for all $i=1, \ldots, p$ and $u$ such that $\|u\| \leq 1$. According to the assumption, we can replace $u=\frac{c_{i}}{\left\|c_{i}\right\|}$, getting $c_{i}^{\prime} d \leq-\delta\left\|c_{i}\right\|<0, i=1, \ldots, p$, and so $f(d)<0_{p}$. Taking $\varepsilon>0$ as in the proof of (i) we obtain

$$
f(\bar{x}+\varepsilon d)=f(\bar{x})+\varepsilon f(d)<f(\bar{x}),
$$

so that $\bar{x} \notin X_{w E}$.

Now, let $D(X ; \bar{x}) \cap \operatorname{int} G^{-}=\emptyset$ for a certain feasible point $\bar{x} \in X$. Suppose that $\bar{x}$ is not a weakly efficient point, i.e., there exists $x \in X$ such that $f(\bar{x})>f(x)$ or, equivalently, $c_{i}^{\prime}(x-\bar{x})<0, i=1, \ldots, p$. Thus, $x-\bar{x} \in D(X ; \bar{x}) \cap$ int $G^{-}$, which completes the proof.

Replacing in Proposition $12 D(X ; \bar{x})$ with bigger and smaller cones (as $A(X ; \bar{x})=T(X ; \bar{x})$ and $T^{i}(X ; \bar{x})$, respectively) one get sufficient and necessary conditions for $\bar{x} \in X_{s E}, \bar{x} \in X_{E}$, and $\bar{x} \in X_{w E}$. The next example shows that the assumption $c_{i} \neq 0, i=1, \ldots, p$, in Proposition 12(iii) is not superfluous.

Example 13 Let $n=p=2, f(x)=\left(0, x_{2}\right)$ and $X=[0,1]^{2}$. Since $f(X)=$ $\{0\} \times[0,1]$ and $G^{-}=\mathbb{R} \times \mathbb{R}_{-}, X_{s E}=\emptyset$ (as $D(X ; \bar{x}) \cap G^{-}$contains halflines for all $\bar{x} \in X), X_{E}=[0,1] \times\{0\}$ (whose elements satisfy $D(X ; \bar{x}) \cap G^{-} \subset \operatorname{ker} f$ ) and $X_{w E}=X$. In particular, if $\bar{x} \in \operatorname{int} X$, we have $D(X ; \bar{x}) \cap \operatorname{int} G^{-}=\mathbb{R} \times$ $\mathbb{R}_{--} \neq \emptyset$, so that statement (iii) fails.

Observe that $\operatorname{dim} G=\operatorname{dim} f\left(\mathbb{R}^{n}\right)$, so that $\operatorname{dim} G+\operatorname{dim} \operatorname{ker} f=n$. In particular, $\operatorname{dim} G=n$ if and only if $\operatorname{ker} f=\left\{0_{n}\right\}$. In that case, $X_{E}=X_{s E}$, and $X_{E}=\operatorname{cl} X_{p E}$ whenever $X_{p E}$ is bounded, by Proposition 12 and [8, Theorem 3.17], respectively. Replacing in Proposition $12 D(X ; \bar{x})$ with the smaller cone $T^{i}(X ; \bar{x})$ one gets necessary conditions for $\bar{x} \in X_{E}, \bar{x} \in X_{E}$, and $\bar{x} \in X_{w E}$.

Example 14 Let

$$
P: " \min " f\left(x_{1}, x_{2}, x_{3}\right)=\left(x_{1}, x_{2}\right) \text { s.t. } a_{t}^{\prime} x \geq b_{t}, t \in T,
$$

be a linear VSIO problem with feasible solution set

$$
X=\text { cone }\left\{\left(x_{1}, x_{2}, 1\right) \in \mathbb{R}^{3}:\left(x_{1}-1\right)^{2}+\left(x_{2}-1\right)^{2}=2\right\} .
$$


Then ker $f=\operatorname{span}\{(0,0,1)\}$ and the image of the closed convex cone $X$ by $f$ is the non-closed convex cone

$$
f(X)=\left\{y \in \mathbb{R}^{2}: y_{1}+y_{2}>0\right\} \cup\left\{0_{2}\right\} .
$$

Taking $\bar{x}=0_{3}$, we have

$$
D(X ; \bar{x}) \cap G^{-}=X \cap\left(\mathbb{R}_{-}^{2} \times \mathbb{R}\right)=\text { cone }\{(0,0,1)\},
$$

so that, by Proposition 12, $\bar{x} \in X_{E} \backslash X_{s E}$ (actually, $X_{E}=X_{w E}=$ cone $\{(0,0,1)\}$ while $X_{s E}=\emptyset$ ).

Corollary 15 Let $P$ satisfy $L O P C Q$ at $\bar{x} \in X$. Then the following statements hold:

(i) $\bar{x} \in X_{s E}$ if and only if $A(\bar{x})^{+} \cap G^{-}=\left\{0_{n}\right\}$ if and only if $A(\bar{x})-G=\mathbb{R}^{n}$.

(ii) $\bar{x} \in X_{E}$ if and only if $A(\bar{x})^{+} \cap G^{-} \subset \operatorname{ker} f$ if and only if $G \subset \operatorname{cl}(A(\bar{x})-G)$.

(iii) Assume that $0_{n} \notin\left\{c_{1}, \ldots, c_{p}\right\}$. Then, $\bar{x} \in X_{w E}$ if and only if $A(\bar{x})^{+} \cap$ $\operatorname{int} G^{-}=\emptyset$.

Proof: The first equivalence in (i) and (ii) derive from Proposition 12 and the fact that $D(X ; \bar{x})=A(\bar{x})^{+}$whenever $P$ satisfies LOPCQ at $\bar{x}$ (the same is true for the equivalence in (iii)). The key of the proof of the second statements in (i) and (ii) is the identity

$$
(A(\bar{x})-G)^{+}=A(\bar{x})^{+} \cap G^{-} .
$$

(i) If $A(\bar{x})^{+} \cap G^{-}=\left\{0_{n}\right\}$, taking positive polars we get $\operatorname{cl}(A(\bar{x})-G)=\mathbb{R}^{n}$, so that $A(\bar{x})-G=\mathbb{R}^{n}$. Conversely, if $A(\bar{x})-G=\mathbb{R}^{n}$, then $A(\bar{x})^{+} \cap G^{-}=\left\{0_{n}\right\}$. So, $A(\bar{x})^{+} \cap G^{-}=\left\{0_{n}\right\} \Leftrightarrow A(\bar{x})-G=\mathbb{R}^{n}$.

(ii) If $A(\bar{x})^{+} \cap G^{-} \subset \operatorname{ker} f$, then $G \subset \operatorname{span}\left\{c_{1}, \ldots, c_{p}\right\}=(\operatorname{ker} f)^{+} \subset \operatorname{cl}(A(\bar{x})-G)$. Conversely, if $G \subset \operatorname{cl}(A(\bar{x})-G)$, then $A(\bar{x})^{+} \cap G^{-} \subset G^{+}$, so that $A(\bar{x})^{+} \cap G^{-} \subset$ $G^{+} \cap G^{-}=\operatorname{ker} f$. Thus, $A(\bar{x})^{+} \cap G^{-} \subset \operatorname{ker} f \Leftrightarrow G \subset \operatorname{cl}(A(\bar{x})-G)$.

Observe that $\bar{x}$ is an optimal solution to the $p$ scalar SIO problems associated with $P$ if and only if $G \subset \operatorname{cl} A(\bar{x})$, which obviously implies that $G \subset$ $\operatorname{cl}(A(\bar{x})-G)$ (the converse is not true). Consider Example 6, where LOPCQ holds at $0_{2}$. Since $G=\mathbb{R}_{+}^{2}$, we have $A\left(0_{2}\right)^{+} \cap G^{-}=X \nsubseteq$ ker $f$ (or, equivalently, $\left.G \nsubseteq \operatorname{cl}\left(A\left(0_{2}\right)-G\right)=A\left(0_{2}\right)-G=A\left(0_{2}\right)\right)$ and $A\left(0_{2}\right)^{+} \cap \operatorname{int} G^{-}=\emptyset$, so that $0_{2} \in X_{w E} \backslash X_{E}$.

Corollary 16 Given $\bar{x} \in X, \bar{x} \in X_{w E}$ if and only if

$$
\left\{d \in \mathbb{R}^{n}: c_{i}^{\prime} d<0, i=1, \ldots, p\right\} \cap D(X ; \bar{x})=\emptyset .
$$

Proof: If $0_{n} \in\left\{c_{1}, \ldots, c_{p}\right\}$, then $\left\{d \in \mathbb{R}^{n}: c_{i}^{\prime} d<0, i=1, \ldots, p\right\}=\emptyset$ and (15) holds trivially. Otherwise, (15) holds by Proposition 12 and the equation $\operatorname{int} G^{-}=\left\{d \in \mathbb{R}^{n}: c_{i}^{\prime} d<0, i=1, \ldots, p\right\}$. The proof of the converse is similar. 
If LOPCQ holds at $\bar{x} \in X_{w E}$, according to (3) and Corollary 16, we get

$$
\left\{d \in \mathbb{R}^{n}: c_{i}^{\prime} d<0, i=1, \ldots, p\right\} \cap T(X ; \bar{x})=\emptyset .
$$

The next lemma, to be used later, shows that (16) is actually a necessary condition for $\bar{x} \in X_{w E}$ in arbitrary linear VSIO problems. This is a well-known result in nonlinear VO (see, e.g., [1, Corollary 3.1], [18, Theorem 2.1], [21, Theorem 7.2] or [27, Theorem 3.1]) whose simple proof is included here for sake of completeness.

Lemma 17 If $\bar{x} \in X_{w E}$, then (16) holds.

Proof: Let $d \in \mathbb{R}^{n}$ be such that $c_{i}^{\prime} d<0, i=1, \ldots, p$. Then, there exists $\varepsilon>0$ such that

$$
c_{i}^{\prime} d<-\varepsilon<0, i=1, \ldots, p .
$$

Suppose that $d \in T(X ; \bar{x})$. Then, there exist sequences $\left\{s^{k}\right\}_{k \in \mathbb{N}}$ and $\left\{d^{k}\right\}_{k \in \mathbb{N}}$ such that $s^{k} \rightarrow 0^{+}, d^{k} \rightarrow d$ as $k \rightarrow \infty$ and $\bar{x}+s^{k} d^{k} \in X$ for all $k \in \mathbb{N}$. Since $d^{k} \rightarrow d$ as $k \rightarrow \infty$, by (17), for $k$ sufficiently large, we have that $c_{i}^{\prime} d^{k}<0$, $i=1, \ldots, p$. Since $c_{i}^{\prime}\left(\bar{x}+s^{k} d^{k}\right)-c_{i}^{\prime} \bar{x}=s^{k} c_{i}^{\prime} d^{k}, i=1, \ldots, p$ and $s^{k}>0$, for $k$ sufficiently large we obtain $c_{i}^{\prime}\left(\bar{x}+s^{k} d^{k}\right)<c_{i}^{\prime} \bar{x}, i=1, \ldots, p$ which contradicts that $\bar{x} \in X_{w E}$.

Proposition 18 Given $\bar{x} \in X$, the following statements hold:

(i) If $\sum_{i=1}^{p} \lambda_{i} c_{i} \in \operatorname{int} D(X ; \bar{x})^{+}$for some $\lambda \geq 0_{p}$, then $\bar{x} \in X_{s E} \cap X_{p E}$.

(ii) $\bar{x} \in X_{p E}$ if and only if $\sum_{i=1}^{p} \lambda_{i} c_{i} \in D(X ; \bar{x})^{+}$for some $\lambda>0_{p}$.

(iii) $\bar{x} \in X_{w E}$ if and only if $\sum_{i=1}^{p} \lambda_{i} c_{i} \in D(X ; \bar{x})^{+}$for some $\lambda \geq 0_{p}$.

Proof. We associate with $P$ the parameterized (weighted) problem

$$
P(\lambda): \min \lambda^{\prime} f(x)=\sum_{i=1}^{p} \lambda_{i} c_{i}^{\prime} x \text { s.t. } x \in X,
$$

where $\lambda \geq 0_{p}$ is the weight vector and $P(\lambda)$ is a linear SIO problem for each $\lambda$ (we could aggregate $\sum_{i=1}^{p} \lambda_{i}=1$ ). Let $\bar{x} \in X$.

(i) Assume that $\sum_{i=1}^{p} \lambda_{i} c_{i} \in \operatorname{int} D(X ; \bar{x})^{+}$for some $\lambda \geq 0_{p}$.

On the one side, according to [13, Theorem 3.1], $\bar{x} \in X$ is the unique optimal solution of $P(\lambda)$ and, by [8, Proposition 3.9], $\bar{x} \in X_{s E}$. 
On the other side, by a continuity argument, there exists $\gamma>0_{p}$ such that $\sum_{i=1}^{p} \gamma_{i} c_{i} \in D(X ; \bar{x})^{+}$and we conclude that $\bar{x} \in X_{p E}$ by statement (ii) (to be proved now).

(ii) According to [13, Lemma 2.3] and the Geoffrion Theorem ([10], see also [8, Theorem 3.15]), there exists $\lambda>0_{p}$ such that $\sum_{i=1}^{p} \lambda_{i} c_{i} \in D(X ; \bar{x})^{+}$if and only if $\bar{x}$ is an optimal solution of $P(\lambda)$ for some $\lambda>0_{p}$ if and only if $\bar{x} \in X_{p E}$.

(iii) Similarly, by [13, Lemma 2.3] and [8, Proposition 3.10], there exists $\lambda \geq 0_{p}$ such that $\sum_{i=1}^{p} \lambda_{i} c_{i} \in D(X ; \bar{x})^{+}$if and only if $\bar{x}$ is an optimal solution of $P(\lambda)$ for some $\lambda \geq 0_{p}$ if and only if $\bar{x} \in X_{w E}$.

Observe that (3) implies that $D(X ; \bar{x})^{+}=A(X ; \bar{x})^{+}=T(X ; \bar{x})^{+}$, so that Proposition 18 remains valid replacing $D(X ; \bar{x})$ with $A(X ; \bar{x})=T(X ; \bar{x})$. Analogously, from $(2), D(X ; \bar{x})$ can also be replaced with $T^{i}(X ; \bar{x})$ whenever the latter cone is non-empty.

Two previous examples allow to show that the sets $X_{s E}$ and $X_{p E}$ are not related by the inclusion. First, take Example 14, with $\bar{x}=0_{2}$. Taking $\lambda=$ $(1,1)>0_{2}$, we have

$$
\lambda_{1} c_{1}+\lambda_{2} c_{2}=(1,1,0) \in \mathbb{R}_{+}^{3} \subset X^{+}=D(X ; \bar{x})^{+} .
$$

Moreover,

$$
\left\{\sum_{i=1}^{2} \lambda_{i} c_{i}: \lambda \geq 0_{2}\right\} \cap \operatorname{int} D(X ; \bar{x})^{+}=\left(\mathbb{R}_{+}^{2} \times\{0\}\right) \backslash\left\{0_{3}\right\} \cap \operatorname{int} X^{+}=\emptyset,
$$

so that $\bar{x} \in X_{p E} \backslash X_{s E}$ by Proposition 18. Now consider Example 2, where $\bar{x}=$ $(1,0)$. The relevant cones are $G=\mathbb{R}_{+}^{2}$ and $D(X ; \bar{x})=\left(\mathbb{R} \times \mathbb{R}_{++}\right) \cup$ cone $\{(1,0)\}$. Since $D(X ; \bar{x}) \cap G^{-}=\left\{0_{2}\right\}$ and

$$
\left\{\sum_{i=1}^{2} \lambda_{i} c_{i}: \lambda>0_{2}\right\} \cap D(X ; \bar{x})^{+}=\mathbb{R}_{++}^{2} \cap\{0\} \times \mathbb{R}_{+}=\emptyset,
$$

$\bar{x} \in X_{s E} \backslash X_{p E}$. The latter example also shows that the converse of Proposition 18(i) is not true. In fact, $\widehat{x}:=\left(\frac{1}{4}, \frac{1}{4}\right) \in X_{p E}$ by Proposition 18(ii), taking $\lambda=(1,1)$, and $\widehat{x} \in X_{s E}$ by Proposition 12(i), while

$$
\operatorname{int} D(X ; \widehat{x})^{+}=\operatorname{int}\left(\left\{d \in \mathbb{R}^{2}: d_{1}+d_{2}>0\right\} \cup\left\{0_{2}\right\}\right)^{+}=\operatorname{int} \text { cone }\{(1,1)\}=\emptyset \text {. }
$$

Corollary 19 If $0_{n} \notin \operatorname{conv}\left\{c_{1}, \ldots, c_{p}\right\}$, then $X_{w E} \subset$ bd $X$.

Proof: Let $\bar{x} \in X_{w E} \cap \operatorname{int} X$. By Proposition 18, there exists $\lambda \geq 0_{p}$ such that $\sum_{i=1}^{p} \lambda_{i} c_{i} \in D(X ; \bar{x})^{+}=\left\{0_{n}\right\}$. Then, $0_{n} \in \operatorname{conv}\left\{c_{1}, \ldots, c_{p}\right\}$. 
Lemma $200_{n} \in \operatorname{conv}\left\{c_{1}, \ldots, c_{p}\right\}$ if and only if $G$ contains lines or some component of $f$ is identically zero.

Proof: If $G$ contains lines, there exists $u \in \mathbb{R}^{n} \backslash\left\{0_{n}\right\}$ such that $\pm u \in G$. Then, we can write $u=\sum_{i=1}^{p} \alpha_{i} c_{i}$ and $-u=\sum_{i=1}^{p} \beta_{i} c_{i}$, with $\alpha_{1}, \ldots, \alpha_{p}, \beta_{1}, \ldots, \beta_{p} \in$ $\mathbb{R}_{+}$, so that

$$
0_{n}=\left(\sum_{i=1}^{p}\left(\alpha_{i}+\beta_{i}\right)\right)^{-1} \sum_{i=1}^{p}\left(\alpha_{i}+\beta_{i}\right) c_{i} \in \operatorname{conv}\left\{c_{1}, \ldots, c_{p}\right\} .
$$

Alternatively, if one component of $f$ is identically zero, then $0_{n} \in\left\{c_{1}, \ldots, c_{p}\right\} \subset$ $\operatorname{conv}\left\{c_{1}, \ldots, c_{p}\right\}$.

Conversely, if $0_{n} \in \operatorname{conv}\left\{c_{1}, \ldots, c_{p}\right\}$, there exist $\alpha_{1}, \ldots, \alpha_{p} \in \mathbb{R}_{+}$such that $\sum_{i=1}^{p} \alpha_{i}=1$. Let $j \in\{1, \ldots, p\}$ be such that $\alpha_{j}>0$. Then the $j$-th component of $f$ is identically zero if $c_{j}=0_{n}$ and $G$ contains the line spanned by $c_{j}$, otherwise.

Proposition 21 If $0_{n} \in \operatorname{conv}\left\{c_{1}, \ldots, c_{p}\right\}$, then $X_{w E}=X$. Otherwise, given $\bar{x} \in X, \bar{x} \in X_{w E}$ if and only if $G \cap D(X, \bar{x})^{+} \neq\left\{0_{n}\right\}$.

Proof: Let $0_{n} \in \operatorname{conv}\left\{c_{1}, \ldots, c_{p}\right\}$. By Lemma 20, either one component of $f$ is identically zero (in which case $X=X_{w E}$ ) or $G$ contains lines. Assume that $G=$ cone $\left\{c_{1}, \ldots, c_{p}\right\}$ is not pointed. Let $\alpha_{1}, \ldots, \alpha_{p} \in \mathbb{R}_{+}$be such that $\sum_{i=1}^{p} \alpha_{i} c_{i}=0_{n}$ and $\sum_{i=1}^{p} \alpha_{i}=1$. If $X \neq X_{w E}$ there exist $\bar{x}, \widehat{x} \in X$ such that $f(\widehat{x})<f(\bar{x})$. Then we get the following contradiction:

$$
0=\left(\sum_{i=1}^{p} \alpha_{i} c_{i}\right)^{\prime}(\widehat{x}-\bar{x})=\sum_{i=1}^{p} \alpha_{i} c_{i}^{\prime}(\widehat{x}-\bar{x})=\sum_{i=1}^{p} \alpha_{i}\left(f_{i}(\widehat{x})-f_{i}(\bar{x})\right)<0 .
$$

So, $X_{w E}=X$.

Now we assume that $\bar{x} \in X$ and $0_{n} \notin \operatorname{conv}\left\{c_{1}, \ldots, c_{p}\right\}$. Then there exists $\lambda \geq 0_{p}$ such that $\sum_{i=1}^{p} \lambda_{i} c_{i} \in D(X ; \bar{x})^{+}$if and only if $\operatorname{conv}\left\{c_{1}, \ldots, c_{p}\right\} \cap$ $D(X, \bar{x})^{+} \neq\left\{0_{n}\right\}$ if and only if $G \cap D(X, \bar{x})^{+} \neq\left\{0_{n}\right\}$. The conclusion follows from Proposition 18(iii).

For instance, in Example 6,

$$
G \cap D\left(X, 0_{2}\right)^{+}=\mathbb{R}_{-} \times \mathbb{R} \cap \mathbb{R}_{+}^{2}=\text { cone }\{(0,1)\} \neq\left\{0_{2}\right\},
$$

so that $0_{2} \in X_{w E}$. The next result is a straightforward consequence of Proposition 21 . 
Corollary 22 Let $0_{n} \notin \operatorname{conv}\left\{c_{1}, \ldots, c_{p}\right\}$ and $\bar{x} \in X$. If $G \cap A(\bar{x}) \neq\left\{0_{n}\right\}$ then $\bar{x} \in X_{w E}$, and the converse holds whenever P satisfies $L F M C Q$ at $\bar{x} \in X$.

Theorem 23 Given $\bar{x} \in X$, the following statements hold:

(i) If there exist $t^{j} \in T(\bar{x}), j=1, \ldots, q \leq n$, and vectors $\lambda=\left(\lambda_{1}, \ldots, \lambda_{p}\right) \geq 0_{p}$ $\left(\lambda>0_{p}\right), \mu=\left(\mu_{1}, \ldots, \mu_{q}\right) \geqq 0_{q}$ satisfying the equation

$$
\text { (KKT) } \quad \sum_{i=1}^{p} \lambda_{i} c_{i}=\sum_{j=1}^{q} \mu_{j} a_{t^{j}}
$$

then $\bar{x} \in X_{w E}\left(\bar{x} \in X_{p E}\right.$, respectively). If, additionally, $\left\{a_{t^{j}}: \mu_{j}>0, j=1, \ldots, q\right\}$ is a basis of $\mathbb{R}^{n}$, then $\bar{x} \in X_{s E} \cap X_{p E}$.

(ii) If $\bar{x} \in X_{w E}\left(\bar{x} \in X_{p E}\right.$ ) satisfies LFMCQ, then there exist $t^{j} \in T(\bar{x})$, $j=1, \ldots, q \leq n$, and vectors $\lambda \geq 0_{p}\left(\lambda>0_{p}\right.$, respectively) and $\mu \geqq 0_{q}$ such that (KKT) holds. If, additionally, $0_{n} \notin \operatorname{conv}\left\{c_{1}, \ldots, c_{p}\right\}$, then the following stronger condition holds:

$$
\sum_{i=1}^{p} \lambda_{i} c_{i}=\sum_{j=1}^{q} \mu_{j} a_{t^{j}} \neq 0_{n}
$$

Proof: (i) Let $t^{j} \in T(\bar{x}), j=1, \ldots, q \leq n$ and $\lambda \in \mathbb{R}^{p}, \mu \geqq 0_{q}$ satisfying (KKT). Then, $\sum_{i=1}^{p} \lambda_{i} c_{i} \in A(\bar{x}) \subset D(X ; \bar{x})^{+}$by (5). So, by Proposition 18 , $\bar{x} \in X_{w E}\left(\bar{x} \in X_{p E}\right)$ whenever $\lambda \geq 0_{p}\left(\lambda>0_{p}\right.$, respectively).

If $\lambda \geq 0_{p}$ and $\left\{a_{t^{j}}: \mu_{j}>0, j=1, \ldots, q\right\}$ is a basis of $\mathbb{R}^{n}$, then we have $\sum_{i=1}^{p} \lambda_{i} c_{i} \in \operatorname{int} A(\bar{x}) \subset \operatorname{int} D(X ; \bar{x})^{+}$and the conclusion follows from Proposition 18(i).

(ii) We are assuming that $A(\bar{x})=D(X ; \bar{x})^{+}$. The first part is straightforward consequence of Proposition 18 while the second one follows from the argument of Proposition 21.

\section{KKT conditions in continuous VSIO}

From now on we assume that $T$ is a compact Hausdorff topological space and all the functions involved in the constraints are continuous on $T$. Under this continuity assumption, if $P$ satisfies SCQ, then FMCQ holds and so, $P$ satisfies LFMCQ at any $\bar{x} \in X$ and (KKT) is a necessary condition for weak efficiency by Theorem 23(ii). Next we prove that this necessary condition still holds whenever $P$ satisfies some weaker c.q. at $\bar{x}$.

Theorem 24 Let $0_{n} \notin \operatorname{conv}\left\{c_{1}, \ldots, c_{p}\right\}$ and $\bar{x} \in X_{w E}$ satisfying EKTCQ and such that $A(\bar{x})$ is closed. Then, there exist $t^{j} \in T(\bar{x}), j=1, \ldots, q \leq n$, as well as vectors $\lambda \geq 0_{p}, \mu \geqq 0_{q}$ satisfying (KKT). 
Proof: $0_{n} \notin \operatorname{conv}\left\{c_{1}, \ldots, c_{p}\right\}$ entails that $\bar{x} \in$ bd $X$ by Corollary 19 while $\bar{x} \in X_{w E}$ implies that

$$
\left\{d \in \mathbb{R}^{n}: c_{i}^{\prime} d<0, i=1, \ldots, p\right\} \cap T(X ; \bar{x})=\emptyset
$$

by Lemma 17. Combining the formulas (8) and (19) with Lemma 11, we conclude that there is no $d \in \mathbb{R}^{n}$ such that

$$
c_{i}^{\prime} d<0, i=1, \ldots, p
$$

and

$$
a_{t}^{\prime} d \geq 0 \text { for all } t \in T(\bar{x}) .
$$

By the Motzkin's Theorem ([12, Theorem 3.5]) (20)-(21) is inconsistent if and only if $0_{n} \in \operatorname{conv}\left\{c_{1}, \ldots, c_{p}\right\}-\operatorname{cone}\left\{a_{t}, t \in T(\bar{x})\right\}$. Thus, we get that there exist $t^{j} \in T(\bar{x}), j=1, \ldots, q \leq n$, as well as vectors $\lambda \geq 0_{p}, \mu \geqq 0_{q}$ such that (KKT) holds.

The following example shows that the closedness assumption in Theorem 24 is not superfluous.

Example 25 Consider the continuous linear VSIO problem

$$
\begin{array}{ccc}
P: & \text { "min " } & f(x)=\left(x_{1}-x_{2},-x_{2}\right) \\
& \text { s.t. } & -t x_{1}-\left(\sqrt{1-(t-1)^{2}}\right) x_{2} \geq 0, t \in[0,2] .
\end{array}
$$

Since

$$
\begin{aligned}
& K=\left(\left\{x \in \mathbb{R}^{2}: x_{1}<0, x_{2} \leq 0\right\} \cup\left\{0_{2}\right\}\right) \times \mathbb{R}_{-}, \\
& \operatorname{cl} K=\text { cone }\{(-1,0,0),(0,-1,0),(0,0,-1)\},
\end{aligned}
$$

and so the feasible set is the cone $X=\mathbb{R}_{-}^{2}$. Moreover,

$$
f(X)=\text { cone }\{f(-1,0), f(0,-1)\}=\text { cone }\{(-1,0),(1,1)\}
$$

and $\bar{x}=0_{2} \in X_{w E}$. Since $T(\bar{x})=[0,2], A(\bar{x})$ is the orthogonal projection of $K$ on the hyperplane $x_{3}=0$, i.e., it is the non-closed convex cone

$$
A(\bar{x})=\left\{x \in \mathbb{R}^{2}: x_{1}<0, x_{2} \leq 0\right\} \cup\left\{0_{2}\right\} .
$$

On the other hand, $A(X ; \bar{x})=\operatorname{cl} A(\bar{x})=X$, and for $d \in \mathbb{R}^{2}$, we have

$$
\varphi^{\prime}(\bar{x}, d) \geq 0 \Leftrightarrow-t d_{1}-\left(\sqrt{1-(t-1)^{2}}\right) d_{2} \geq 0 \text { for all } t \in[0,2] .
$$

From the latter inequality it follows that $\left\{d \in \mathbb{R}^{2}: \varphi^{\prime}(\bar{x}, d) \geq 0\right\}=X$. Therefore, EKTCQ is fulfilled. Now, consider the condition

$$
\lambda_{1}\left(\begin{array}{c}
1 \\
-1
\end{array}\right)+\lambda_{2}\left(\begin{array}{c}
0 \\
-1
\end{array}\right)=\sum_{i=1}^{2} \mu_{i}\left(\begin{array}{c}
-t^{i} \\
-\sqrt{1-\left(t^{i}-1\right)^{2}}
\end{array}\right)
$$




$$
\mu_{i} \geq 0, t^{i} \in[0,2], i=1,2 .
$$

A few calculations show that (22)-(23) is fulfilled if and only if $\lambda_{1}=\lambda_{2}=\mu_{1}=$ $\mu_{2}=0$. Altogether, we conclude that (KKT) cannot be fulfilled.

Corollary 26 Let $0_{n} \notin \operatorname{conv}\left\{c_{1}, \ldots, c_{p}\right\}$ and $\bar{x} \in X_{w E}$ satisfying LSCQ. Then, there exist $t^{j} \in T(\bar{x}), j=1, \ldots, q \leq n$, as well as vectors $\lambda \geq 0_{p}, \mu \geqq 0_{q}$ satisfying (KKT).

Proof: $0_{n} \notin \operatorname{conv}\left\{c_{1}, \ldots, c_{p}\right\}$ entails that $\bar{x} \in$ bd $X$ by Corollary 19 . Then, $P$ satisfies EKTCQ at $\bar{x}$ by Lemma 9 . Since $A(\bar{x})$ is closed by Lemma 5 , the conclusion follows from Theorem 24 .

The next definition adapts, to continuous linear VSIO, another concept of proper efficiency which was introduced by H. Kuhn and A. Tucker (cf. [19]) in the framework of standard vector optimization problems: $x \in X$ is called properly efficient in Kuhn-Tucker sense for $P$ if $x \in X_{E}$ and the system

$$
\left\{f(d) \leq 0_{p}, \varphi^{\prime}(x, d) \geq 0\right\}
$$

has no solution in $\mathbb{R}^{n}$. We denote by $X_{p E}^{k t}$ the set of properly efficient points in Kuhn-Tucker sense.

Intuitively, the existence of solution $d$ to the system (24) means that moving from $x$ in direction $d$ no objective function increases, one strictly decreases, and the cone $C:=\left\{d \in \mathbb{R}^{n}: \varphi^{\prime}(x, d) \leq 0\right\}$ is not left. Thus, $d$ is a direction of "descent" in the cone $C$. For instance, in Example 2, where $\bar{x}=(1,0) \in$ $X_{s E} \backslash X_{p E}$, we have

$$
\left\{d \in \mathbb{R}^{2}: f(d) \leq 0_{2}, \varphi^{\prime}(\bar{x}, d) \geq 0\right\}=\text { cone }\{(-1,0)\} \backslash\left\{0_{2}\right\},
$$

so that $\bar{x} \notin X_{p E}^{k t}$. The vector $d=(-1,0) \in C$ is a descent direction for the first objective while it is a stationary direction for the second one.

In [8, Theorem 2.51] and [26, Theorem 3.1.6] it is proved, for standard VO (i.e., with $T$ finite), that under EKTCQ, $x \in X_{p E}$ implies $x \in X_{p E}^{k t}$. The next theorem shows that this statement still holds for linear VSIO problems. We include the proof (essentially the same as in standard VO) for sake of completeness.

Theorem 27 If $\bar{x} \in X_{p E}$ satisfies EKTCQ, then $\bar{x} \in X_{p E}^{k t}$.

Proof: Suppose that $\bar{x} \in X_{E} \backslash X_{p E}^{k t}$. Then, there is some $d \in \mathbb{R}^{n}$ such that (without loss of generality, reordering the objectives if it is necessary)

$$
\begin{gathered}
c_{1}^{\prime} d<0, \\
c_{k}^{\prime} d \leq 0, k=2, \ldots, p,
\end{gathered}
$$

and

$$
\varphi^{\prime}(\bar{x}, d) \geq 0 .
$$


By $(27),(3)$ and the EKTCQ condition, $d \in A(X ; \bar{x})=T(X ; \bar{x})$. Therefore, there exist sequences $\left\{s^{k}\right\}_{k \in \mathbb{N}}$ and $\left\{d^{k}\right\}_{k \in \mathbb{N}}$ such that $s^{k} \rightarrow 0^{+}, d^{k} \rightarrow d$ as $k \rightarrow \infty$ and $\bar{x}+s^{k} d^{k} \in X$ for all $k \in \mathbb{N}$. It holds that, for a subsequence $\left\{s^{k_{r}}\right\}_{r \in \mathbb{N}}$ of $\left\{s^{k}\right\}_{k \in \mathbb{N}}$ the set

$$
I=\left\{i: c_{i}^{\prime}\left(\bar{x}+s^{k_{r}} d^{k_{r}}\right)>c_{i}^{\prime} \bar{x}\right\}
$$

is the same for all $r$. For simplicity in notations assume that

$$
I=\left\{i: c_{i}^{\prime}\left(\bar{x}+s^{k} d^{k}\right)>c_{i}^{\prime} \bar{x}\right\}
$$

for all $k \in \mathbb{N}$. Now, for $i \in I$, we have

$$
c_{i}^{\prime}\left(\bar{x}+s^{k} d^{k}\right)-c_{i}^{\prime} \bar{x}=s^{k} c_{i}^{\prime} d^{k}>0 .
$$

Dividing by $s^{k}$ and taking limit in (28), as $k \rightarrow \infty$ it follows that

$$
0 \leq c_{i}^{\prime} d \leq 0
$$

Therefore

$$
c_{i}^{\prime} d=0 \text { for all } i \in I .
$$

Note that $1 \notin I$ by (25). Finally, since $c_{1}^{\prime} d<0$ the condition (29) implies (for $i \in I$ ) that

$$
\frac{c_{1}^{\prime} \bar{x}-c_{1}^{\prime}\left(\bar{x}+s^{k} d^{k}\right)}{c_{i}^{\prime}\left(\bar{x}+s^{k} d^{k}\right)-c_{i}^{\prime} \bar{x}}=\frac{-c_{1}^{\prime} d^{k}}{c_{i}^{\prime} d^{k}} \rightarrow+\infty .
$$

Hence $\bar{x} \notin X_{p E}$.

The next results give necessary KKT conditions for proper efficiency in Kuhn and Tucker's sense.

Theorem 28 If $\bar{x} \in X_{p E}^{k t}$ and $A(\bar{x})$ is finitely generated, then there exist $t^{j} \in$ $T(\bar{x}), j=1, \ldots, q \leq n$, as well as vectors $\lambda>0_{p}, \mu \geqq 0$ satisfying (KKT).

Proof: Since $\bar{x} \in X_{p E}^{k t}$, there is no $d \in \mathbb{R}^{n}$ satisfying $\left(c_{1}^{\prime} d, \ldots, c_{p}^{\prime} d\right) \leq 0_{p}$ and $\varphi^{\prime}(\bar{x}, d) \geq 0$.

Take $i=1$. The linear inequality $c_{1}^{\prime} d \geq 0$ is consequence of the system

$$
\left\{c_{k}^{\prime} d \leq 0, k=2, \ldots, p ; a_{t}^{\prime} d \geq 0, t \in T(\bar{x})\right\} .
$$

Then, applying (4), $c_{1} \in$ cone $\left\{-c_{2}, \ldots,-c_{p} ; a_{t}, t \in T(\bar{x})\right\}$. Let $\lambda_{2}^{1} \geq 0, \ldots, \lambda_{p}^{2} \geq 0$ and $\mu_{t}^{1} \geq 0, t \in T(\bar{x})$, be such that $c_{1}=-\sum_{i=2}^{p} \lambda_{i}^{1} c_{i}+\sum_{t \in T(\bar{x})} \mu_{t}^{1} a_{t}$. Defining $\lambda_{1}^{1}=1$, we have $\lambda^{1}=\left(\lambda_{1}^{1}, \ldots, \lambda_{p}^{1}\right) \geqq 0_{p}$ such that $\lambda_{1}^{1}>0$ and $\sum_{i=1}^{p} \lambda_{i}^{1} c_{i} \in A(\bar{x})$. The same argument shows the existence of $\lambda^{j}=\left(\lambda_{1}^{j}, \ldots, \lambda_{p}^{j}\right) \geqq 0_{p}$ such that $\lambda_{j}^{j}>0$ and $\sum_{i=1}^{p} \lambda_{i}^{j} c_{i} \in A(\bar{x})$. Hence, the vector $\lambda:=\sum_{j=1}^{p} \lambda^{j}>0_{p}$ satisfies $\sum_{i=1}^{p} \lambda_{i} c_{i} \in A(\bar{x})$. The conclusion follows from Carathéodory's Theorem. 
Corollary 29 If $\bar{x} \in X_{p E}$, satisfies $E K T C Q$, and $A(\bar{x})$ is finitely generated, then there exist $t^{j} \in T(\bar{x}), j=1, \ldots, q \leq n$, as well as vectors $\lambda>0_{p}, \mu \geqq 0$ satisfying (KKT).

Proof: Combine Theorem 27 with Theorem 28.

Corollary 30 Suppose that $\bar{x} \in X_{p E}^{k t}$ and $A(\bar{x})$ is finitely generated. Then $\bar{x} \in X_{p E}$.

Proof: The result follows from Theorem 28 and Theorem 23.

Theorem 31 Let $\bar{x} \in X$ satisfy EKTCQ and assume that there exist $t^{j} \in$ $T(\bar{x}), j=1, \ldots, q \leq n$, as well as vectors $\lambda>0_{p}, \mu \geqq 0$ satisfying (KKT). Then $\bar{x} \in X_{p E}^{k t}$.

Proof: The result follows from Theorems 27 and 23.

Acknowledgement. The authors wish to thank Prof. V. Novo and Dr. B. Jiménez, and also to the unknown referees, whose valuable comments and suggestions have contributed to improve the final presentation of this paper.

\section{References}

[1] Bigi, G. Castellani, M. (2000). Second order optimality conditions for differentiable multiobjective problems, RAIRO Oper. Res. 34, 411-426.

[2] Bonnans, F., \& Shapiro, A. (2000). Perturbation analysis of optimization problems. New York: Springer-Verlag.

[3] Bot, R.I., Grad, S.-M., \& Wanka, G. (2009). Duality in vector optimization. Berlin: Springer-Verlag.

[4] Caristi, G., Ferrara, M., \& Stefanescu, A. (2010). Semi-infinite multiobjective programming with generalized invexity. Math. Reports, 12, 217-233.

[5] Chuong, T.D. (2012) Lower semicontinuity of the Pareto solution map in quasiconvex semi-infinite vector optimization. J. Math. Anal. Appl., 388, 443-450.

[6] Chuong, T.D., Huy, N.Q., \& Yao, J.C. (2009). Stability of semi-infinite vector optimization problems under functional perturbations. J. Global Optim., 45, 583-595.

[7] Chuong, T.D., Huy, N.Q., \& Yao, J.C. (2010). Pseudo-Lipschitz property of linear semi-infinite vector optimization problems. Eur. J. Oper. Res., 200, 639-644.

[8] Ehrgott, M. (2005). Multicriteria optimization (2nd ed). Berlin: SpringerVerlag. 
[9] Fan, X., Cheng, C., \& Wang, H. (2012) Density of stable semi-infinite vector optimization problems under functional perturbations. Oper. Res. Letters, 40, 140-143.

[10] Geoffrion, A. (1968). Proper efficiency and the theory of vector maximization. J. Math. Anal. Appl., 22, 618-630.

[11] Giorgi, G., Jiménez, B., Novo, V. (2004). On constraint qualifications in directionally differentiable multiobjective optimization problems. RAIRO Oper. Res., 38, 255-274.

[12] Goberna, M.A., \& López, M.A. (1998). Linear semi-infinite optimization. Chichester: Wiley.

[13] Goberna, M.A., López, M.A., \& Todorov, M.I. (1995). Unicity in linear optimization. J. Opim. Theory Appl., 86, 37-56.

[14] Guerra, F., Orozco, J.A., \& Rückmann, J.-J. (2004). On constraint qualifications in semi-infinite optimization. In J. Guddat, H. Th. Jongen, J.-J, Rückmann \& M. Todorov (Eds.), Parametric optimization and related topics VII (pp. 133-141), México: Soc. Mat. Mexicana.

[15] Hantoute, A., \& López, M A. (2008). A complete characterization of the subdifferential set of the supremum of an arbitrary family of convex functions. J. Convex Anal., 15, 831-858.

[16] Hartley, R. (1978). On cone-efficiency, cone-convexity and conecompactness. SIAM J. Appl. Math., 34, 211-222.

[17] Helbig, S., \& Todorov,M.I. (1998). Unicity results for general linear semiinfinite optimization problems using a new concept of active constraints. Appl. Math. Optim., 38, 21-43.

[18] Jiménez, B., Novo, V. (2003). Optimality conditions in directionally differentiable Pareto problems with a set constraint via tangent cones. Numer. Funct. Anal. Optim., 24, 557-574.

[19] Kuhn, H., \& Tucker, A. (1951). Nonlinear programming. In J. Newman (Ed.), Proceedings of the second Berkeley symposium on mathematical statistics and probability (pp. 481-492), Berkeley: University of California Press.

[20] Laurent, P.J. (1972). Approximation et optimisation. Paris: Hermann.

[21] Lin, J.G. (1976). Maximal vectors and multi-objective optimization. J. Optimization Theory Appl., 18, 41-64.

[22] López, M.A., \& Volle, M. (2011). On the subdifferential of the supremum of an arbitrary family of extended real-valued functions. Rev. R. Acad. Cienc. Exactas Fís. Nat. Ser. A Math. RACSAM, 105, 3-21. 
[23] Maeda, T. (1994). Constraint qualifications in multiobjective optimization problems: differentiable case. J. Optim. Theory Appl., 80, 483-500.

[24] Mordukhovich, B., \& Nghia, T.T.A. Constraint qualifications and optimality conditions for nonconvex semi-infinite and infinite programs. Math. Programming, to appear.

[25] Peterson, D.W. (1973). A review of constraint qualifications in finitedimensional spaces. SIAM Rev., 15, 639-654.

[26] Sawaragi, Y., Nakayama, Hi., Tanino, T. (1985). Theory of multiobjective optimization. Orlando: Academic Press.

[27] Singh, C (1976). Optimality conditions in multiobjective differentiable programming. J. Optim. Theory Appl., 53, 115-123.

[28] Stein, O. (2001). First-order optimality conditions for degenerate index sets in generalized semi-infinite optimization. Math. Oper. Res., 26, 565-582.

[29] Tapia, R.A., \& Trosset, M.W. (1994). An extension of the Karush-KuhnTucker necessity conditions to infinite programming. SIAM Rev. 36, 1-17.

[30] Todorov, M.I. (1989). Uniqueness of the saddle points for most of the Lagrange functions of the linear semi-infinite optimization. Numer. Funct. Anal. Optim., 10, 367-382.

[31] Todorov, M.I. (1994). Well-posedness in the linear vector semi-infinite optimization. In G.H. Tzeng (Ed.), Multiple criteria decision making (pp. 141-150), New York: Springer-Verlag.

[32] Todorov, M.I. (1996). Kuratowksi convergence of the efficient sets in the parametric linear vector semi-infinite optimization. Eur. J. Oper. Res., 94, 610-617.

[33] Todorov, M.I. (1998). Necessary and sufficient conditions for global lower semicontinuity in linear semi-infinite optimization, Numer. Funct. Anal. Optim., 19, 415-429.

[34] Zălinescu, C. (2002). Convex analysis in general vector spaces. New Jersey: World Scientific Publishing Co. 